\title{
A novel cross-layer mesh router placement scheme for wireless mesh networks
}

\author{
Tein-Yaw Chung ${ }^{1 *}$, Hao-Chieh Chang ${ }^{1}$ and Hsiao-Chih George Lee ${ }^{2}$
}

\begin{abstract}
Wireless mesh networks (WMNs) offer a great promise in supporting ubiquitous multimedia Internet access for mobile or fixed mesh clients (MCs). In WMNs, Internet traffic from MCs is aggregated by serving mesh router (MR) and forwarded hop-by-hop by MRs to an internet gateway (IGW) or vice versa. While deploying MRs and IGWs, intricate relationships among antenna types, wireless links with adaptive modulation and coding, MAC scheduling, routing, and equipment cost render the network planning an extremely complex problem. This article presents a novel cross-layer MR placement (CMRP) scheme that can cope up with this issue. CMRP encapsulates the crosslayer metrics into three underlying attributes: Local Coverage (LC), Backbone Residual Capacity (BRC), and Deployment Cost (DC), and are used to minimize the network deployment cost. Coupled with our proposed novel tree-based minimal cost routing scheme and weight-based link assignment for user coverage, we are able to plan the design of WMNs efficiently. Extensive simulations have been performed to examine the performance and feasibility of CMRP and compared with existing design schemes based on coverage, connectivity, and combination of the two. The result demonstrates that our approach outperforms existing schemes both in the cost performance ratio and potential implementation feasibility.
\end{abstract}

Keywords: capacity improvement, gateway placement, multi-hop relay networks, relay node placement, wireless mesh networks, wireless multi-hop networks

\section{Introduction}

In the near future, broadband wireless mesh networks (WMNs) [1,2] are expected to be widely deployed for providing Internet connectivity to users in residential areas and offices and supplementing existing wired infrastructure. WMNs are characterized by self-organizing and self-configuring capabilities, and hence are easy to be deployed. In $3 \mathrm{G}$ and Wi-Fi networks, each access point (AP) is connected through extensive wired infrastructure to access the backhaul network, which is often expensive and time consuming to be built. On the other hand, WMNs only use a subset of APs, called internet gateways (IGWs), to access the wired network, while the rest of the APs, called mesh routers (MRs), are connected to the IGWs in multi-hop wireless fashion. Thus, they are easy to be built and can provide an economical alternative to broadband wireless Internet connectivity.

\footnotetext{
* Correspondence: csdchung@saturn.yzu.edu.tw

${ }^{1}$ Yuan-Ze University, Chung-Li, Tao-Yuan, Taiwan

Full list of author information is available at the end of the article
}

Although WMN products are available in the market [3-6], their deployment has faced tremendous challenges $[1,2]$ because of some inherent problems, such as interference and high bit error rate (BER). One of the biggest challenge in deploying a WMN is to meet users' requirements with minimal cost. Usually, we have only a limited number of selected places that may have ac power and many locations may not be appropriate for MR deployment. Thus, the problem is to choose some of the locations for MR deployment so as to achieve the best cost performance ratio (CPR). A good location of MRs not only can provide high network throughput but also can lead to minimum number of MRs for meeting users' demand in the WMN design.

In the past, various schemes in various layers [7-17] have been used in placing MRs and IGWs so as to enhance the performance. Intricate relationships among antenna type used, wireless links with adaptive modulation and coding (AMC) scheme, MAC scheduling, routing, and equipment cost render the problem of optimal WMN planning extremely complex to address. Similar to 
the IEEE 802.16j scheme, some researchers [7,8] develop schemes to place MRs so as to improve the WMN throughput, while others discuss the problem of MR placement (MRP) either without considering the wireless backbone network specifications for users' demand [9] or just focusing on the user coverage while ignoring users' demand [10,12-16], not to mention the wireless backbone network support. The authors in [11] present an MRP algorithm without considering the cost for various antenna types. To simplify the problem, these works only consider a part of the design parameters associated with the MRP. Therefore, a more sophisticated MRP scheme is desirable to design a cost effective WMN that can meet users' demand both at the local level and at the backbone, with various technical options, such as antenna types, MAC scheduling, and routing.

This article proposes a cross-layer MRP (CMRP) scheme for a comprehensive MRP problem. Many researchers have proved the cross-layer approach [18-21] to be an effective scheme in improving the network performance. Our new CMRP iteratively adjusts the user coverage by each MR while new MRs are being added. As the residual backbone capacity is being evaluated with respect to the incurred interference, additional demand can be satisfied by each newly added MR. Based on a minimal interference routing scheme and the concept of bottleneck collision domain (BCD) [22], the backbone capacity is also evaluated to see if it can really meet the users' demand. To design a WMN with minimal cost, CMRP deploys a pair of directional antennas whenever it is observed to be cost effective. Therefore, CMRP offers a powerful MR deployment scheme in planning a WMN.

In CMRP, the cross-layer metrics are encapsulated into three attributes: Local Coverage (LC), Backbone Residual Capacity (BRC), and Deployment Cost (DC), which are evaluated throughout the MR addition process. $L C$ specifies the contribution of a new MR in offering additional access capacity to the local users, $B R C$ indicates the contribution of MRs to the backhaul capacity, and $D C$ represents the ratio of the cost of deploying an MR using directional antennas to the cost of deploying an MR using an omni-directional antenna. $D C$ enables selection of antenna types, such as omni-directional or directional antenna, based on the CPR while a WMN is being planned. CPR is taken as the ratio of the total deployment cost to the aggregate throughput in IGW. To maximize the objective function $(L C \times B R C / D C)$, CMRP selects MRs one by one among all the MR candidate locations. In this way, the objective function picks MR candidate locations that largely adds to the backbone capacity, more users' demand coverage, and lower deployment cost.

Extensive simulations have been performed to examine the performance and feasibility of our approach. We also compare CMRP with existing WMN planning schemes that consider only either coverage, or connectivity, or a combination of both. The result illustrates that CMRP outperforms existing schemes both in terms of CPR and its deployment feasibility. In addition, CMRP can help determine the users' demand and the size of a WMN that can achieve the best CPR. This information can help in deciding how many IGWs are needed when a large WMN is being planned.

The remainder of this article is organized as follows. Section 2 describes the related work. Section 3 presents the network model and problem formulation. Section 4 describes our heuristic algorithm. Section 5 summarizes the simulation results. Finally, Section 6 concludes the article and discusses our future work.

\section{Related work}

The inherent drawbacks of WMNs, such as interference, power limitation, and high BER, significantly limit the performance of WMNs. In the past, many researches $[18-21,23,24]$ have presented algorithms to improve the throughput in channel utilization, radio power setting, and time slots allocation of WMNs. However, these works do not consider the service point placement problem, which has been experimentally shown to have a great impact on the performance by Bicket et al. [25].

The service point can be divided into two types of placement: IGW and MRs. The IGW placement [26-33] focuses on the wide area WMN planning, wherein many service points are clustered, and an IGW is assigned to each cluster. The MRP [7-17] deploys MRs to cover all users' demand. MRs may interfere with one another. Thus, if one of the MR wants to improve its throughput or service range using power control, then the nearby MRs may adversely suffer serious interference. So, how to optimize the MRP is an important problem that dictates the overall performance in a WMN system. In this article, we only consider the MRP, while the IGW placement is left as our future work.

So and Liang [7] place a fixed number of tetherless relay points (TRPs) to improve the throughput of a wireless LAN. They present a rate adaptation scheme to estimate the link rate and analyze how various parameters, such as path loss exponent, power ratio of AP and TRP over the power of mobile host $(\mathrm{MH})$, and the number of TRPs, affect the performance and TRP placement. Lin et al. [8] analyze the placement of a single relay node $(\mathrm{RN})$ in the IEEE 802.16j point-to-multi-point (PMP) networks so as to extend the coverage and improve the throughput/capacity of the network. They use a cooperative relay strategy to improve spatial diversity. Wang et al. [9] use a distributed clustering scheme to place a minimum number of MRs on candidate locations. Although they ensure connectivity between MRs, users' demand and users' coverage are met. But, they do not consider the link scheduling at 
the WMN backbone, and thus they cannot guarantee users' demand to be supported by the wireless backbone. San and Raman [10] define a complex objective function to minimize the total cost of MR deployment. Their design considers the number of antennas, the type of antenna, and the height of the IGW which do affect the line-of-sight transmission. Although they have considered the user coverage and the interference problem, they do not take users' demand into account. Moreover, they limit their design to only two-hop networks.

To cover users' need, So and Liang [11] address the MRP problem by constructing a fixed power of local and backbone links. However, they do not consider the cost of different types of antennas. Robinson and Kinghtly [12] analyze the throughput of WMNs with various types of topology, such as triangle, rectangle, hexagon, and random, and then compare the coverage performance. But, they only consider the users' coverage, not the users' demand. Franklin and Murphy [13] consider both the network backbone connectivity and the local coverage problem and use signal strength to represent the connectivity. But, they do not incorporate users' demand, which limits the usefulness of their approach. Xhafa et al. [14-16] use several search methods to solve the MRP problem. They take both the network connectivity and the user coverage. However, they do not consider users' demand, antenna cost, link capacity, or interference issues. Wang et al. [17] minimize the number of MRs deployed, with the objectives of the network connectivity, the users coverage, and users' demand. They consider MRs with multiple rates, which influence both the transmission range and the link capacity. However, they consider only fixed number of antennas on an MR and thus fixed cost per MR.

Selecting network service points with the minimum cost is a challenging task. Although the above researches have worked on this issue, they do not consider comprehensive metrics, such as users' demand, signal interference, MR deployment cost, and antenna type. This article presents a CMRP scheme to minimize the cost of MR deployment by taking users' demand, MAC scheduling, routing, and costs of MR and antenna into consideration.

\section{MRP Modeling and solution}

This article focuses on IEEE 802.16d-based WMNs, in which a set of MRs are connected with multi-hop wireless links to form a wireless backbone, which is then connected to the Internet through an IGW.

\subsection{Network model}

Given $n$ randomly generated user locations $V=\left[v_{1}, \ldots\right.$, $\left.v_{n}\right]$, and $m$ randomly generated MR candidate locations $V^{\prime}=\left[v_{1}^{\prime}, \ldots, v_{m}^{\prime}\right]$, according to the IEEE 802.16d mesh networking standard, assume that all the MC nodes are fixed and only one IGW is selected from these candidate locations. Assume that the user locations and the MR candidate locations satisfy the geographic and RF constraints.

We assume that the 802.16 OFDM modulation scheme is used between an MR and its local MCs. Thus, each MR employs omni-directional antenna for serving its local MCs. We assume the presence of single channel modulation scheme for the backbone. Thus, an MR uses either omni-directional or directional antenna for the backbone of the WMN. A directional antenna (also called a sectored antenna) is different from an omni-directional antenna in that it only transmits the signal in the range of a sector. Because it can concentrate on transmitting power in only a given direction, it can cover a longer range while the interference is limited to a smaller area than that of an omni-directional antenna. Let $P l$ be the maximum power of all the antennas used in the local network, and $P O$ and $P d$, respectively, be the maximum power of an omni-directional and a directional antenna used in the backbone network. In general, the local service antenna has a smaller transmission range, and the backbone service antenna has a larger transmission range, i.e., $P_{L}<P_{O}<P_{D}$.

In the MAC layer, we assume the TDMA scheme as specified by the $802.16 \mathrm{~d}$ mesh mode, and is used for both the local and the backbone WMNs, and the link rate is set by the AMC scheme. In the TDMA scheme, time is partitioned into synchronized frames, which are composed of several equal duration time slots. Links are scheduled to maximize spatial reuse of the link bandwidth while avoiding any collision.

In a WMN, every MR aggregates traffic load from the local MCs. Then, the traffic is relayed between MRs in a multi-hop wireless fashion. As MCs do not communicate directly with each other, MRs form the backbone of a WMN. Unlike ad hoc networks where traffic is randomly distributed between peer nodes, the traffic in a WMN is predominantly directed from MRs toward IGW or from IGW to MRs, i.e., so-called internetwork traffic. We assume that every MC $i$ has a maximum internetwork demand $q_{i}$. We consider maximum users' demand because the ultimate goal of network planning is to satisfy whatever a user may need. We also assume that a symmetric scheme is used in the transmission system, i. e., both downlink and uplink flows interfere in the same way. Thus, we only consider uplink flow demand, as it is easy to extend the system to the downlink flow demand.

\subsection{MRP problem}

In this subsection, we define the MRP problem as a cost minimization problem. Given internetwork demand $q_{i}$, $\forall i \in V$, MR candidate locations $V$, and the price of an MR and the price of a pair of directional antennas, the goal of MRP is to deploy MRs and directional antennas 
to meet users' traffic demand with minimum cost. Assume that the default cost of an MR includes two omni-directional antennas: one for local traffic and another for backbone traffic. The MRP problem can be defined as a mixed integer linear programming (MILP) as follows when a routing tree rooted on IGW is employed.

$$
\min \alpha \sum_{j=1}^{m} x_{j}+\beta \sum_{j=1}^{m} x_{j} y_{j}
$$

with the conditions:

$$
\begin{aligned}
& \sum_{j=1}^{m} z_{i j}=1 \quad \forall i \in V, j \in V^{\prime}, \\
& q_{i} \leq r_{i j} \leq C_{\max } \quad \forall i \in V, j \in V^{\prime}, \\
& \sum_{i=1}^{n} q_{i} z_{i j}=R_{j} \leq R_{\max } \quad \forall j \in V^{\prime}
\end{aligned}
$$

and

$$
\hat{Q}_{k}+\hat{C}_{k}^{\prime} \leq \hat{C}_{k} \leq \hat{C}_{\max } \quad \forall k \in V^{\prime},
$$

where

$\alpha$ : cost of an MR using omni-directional antenna;

$\beta$ : cost increase of an MR using directional antennas;

$x_{j}=\left\{\begin{array}{l}1 \text { if an MR is installed at position } j, \\ 0 \text { otherwise; }\end{array}\right.$

$y_{j}=\left\{\begin{array}{l}1 \text { if a directional antenna is used by MR } j, \\ 0 \text { otherwise; }\end{array}\right.$

$z_{i j}=\left\{\begin{array}{l}1 \text { if user } i \text { is served by MR } j, \\ 0 \text { otherwise; }\end{array}\right.$

$q_{\mathrm{i}}$ : maximum traffic demand of user $i$;

$r_{i j}$ : transmission rate between user $i$ and MR $j$;

$C_{\text {max }}$ : maximum link capacity of a local access antenna;

$R_{\mathrm{j}}$ : local coverage of $\mathrm{MR} j$;

$R_{\text {max }}$ : maximum local coverage of an MR;

$\hat{Q}_{k}$ : aggregate backbone traffic of MR $k$, where

$\hat{Q}_{k}=\sum_{j=1}^{m} \hat{Q}_{j} h_{j k}+Q_{k}$

$h_{j k}=\left\{\begin{array}{l}1 \text { if the traffic of MR } j \text { goes through MR } k \\ 0 \text { otherwise; }\end{array}\right.$
$Q_{k}$ : locally generated traffic of MR $k$;

$\hat{C}_{k}^{\prime}$ : wasted capacity of MR $k$ because of interference from other MRs, where

$\hat{C}_{k}^{\prime}=\left(\sum_{l_{i j} \in \Gamma_{k}} \frac{\hat{Q}_{i}}{r_{i j}} h_{i j}\right) \cdot r_{k l} \cdot h_{k l}$,

where $\Gamma_{k}$ is the set of links that interfere MR $k$ and MR $l$ is the uplink MR of MR $k$;

$\hat{C}_{k}$ : backbone uplink capacity ofaggregate backbone traffic MR $k$;

$\hat{C}_{\text {max }}$ : maximum backbone link capacity of a backbone access antenna with AMC.

Equation 1 is our objective function that minimizes the total cost of MRs and additional directional antennas deployed. Equations 2 and 3 guarantee that each MC $i$ can be served by one MR, and its demand $q_{i}$ can be supported by the transmission rate $r_{i j}$ that is smaller than the maximum link capacity $C_{L}$ with AMC between MC $i$ and MR $j$. Equation 4 guarantees that all locally generated users' demand could be fully covered by the MRs deployed. Equation 5 guarantees that every MR $\mathrm{j}$ can relay inter-MR traffic and support locally generated traffic through its backbone capacity $\hat{C}_{k}$ with interference from other nearby MRs. This constraint of Equation 5 is highly related to the locations of MRs, how a routing path is selected for relaying traffic between MRs and IGW, and the MAC layer scheduling with spatial reuse constraint. $\hat{C}_{k}$, the uplink capacity of MR $k$, is determined by the distance between MR $k$ and its uplink MR based on AMC. As expressed in Equation 6, $\hat{Q}_{k}$, the aggregate traffic of MR k, i.e., the sum of all the transit traffic and locally generated traffic, depends on the routing algorithm, which determines the value of $h_{j k}$. Finally, $\hat{C}_{k}^{\prime}$, the wasted capacity of MR $k$, is determined by the MAC layer scheduling scheme based on the spatial reuse according to the routing tree constructed by the routing algorithm.

The MRP problem as defined in Equation 1 is a crosslayer design problem, which involves equipment cost, antenna type used, wireless AMC, network routing and MAC scheduling. Such an interrelated MILP problem is NP hard [17]. This motivates us to find an effective approach to handle this problem.

In order to solve the MRP problem, we use three novel performance metrics to capture the multi-layer design consideration for the local network and the backbone network: Local Coverage (LC), Backbone Residual Capacity (BRC), and Deployment Cost (DC). LC denotes the users' demand that can be covered by an MR with 
an AMC wireless link, which can be used to evaluate the contribution of an MR to fulfill Equation 4. BRC calculates the residual backbone capacity that can support more users' demand originated from a newly deployed MR. Since the internetwork traffic must be routed hopby-hop to the IGW, it consumes bandwidth of many links and cause interference among links. BRC captures the effect of Equation 5 as it considers the synergy effect of AMC, MAC scheduling, and routing because the chosen location for placing an MR determines the link rate with $A M C$, while the routing path between the MR and IGW consumes the capacities of the path links, which further interferes with links in its neighborhood, and thus the MAC layer must schedule the links to prevent transmission collision. $D C$ can help us evaluate the tradeoff between using directional antennas that increase the backbone capacity or just deploying an MR using omni-directional antenna to save cost while deploying MR. It provides us a vehicle to optimize the cost of the MRP problem indicated in Equation 1.

With these three metrics, we develop a heuristic algorithm to resolve the MRP. First, given a user demand vector, we can use some existing IGW selection scheme, such as the one given in [9], to place an IGW at one of MR candidate locations. Second, with or without directional antennas, we deploy an MR at a selected location with a maximal utility value. Then, we check if all users' demand have been met. If not, add an additional MR that can meet the residual users' demand. The process is repeated until either all users' demand is met or the algorithm fails.

\subsection{Cross-layer design}

Our cross-layer design contains two major parts: the local network and the backbone network. In the local network, we try to satisfy all local users' demand with a minimal number of MRs. In the backbone network, we must ensure all the MRs have sufficient bandwidth to forward their traffic hop-by-hop to the IGW through a MAC scheduling algorithm and a good routing tree. This subsection first discusses the AMC model in the physical layer and a tree-based minimum cost routing (TMCR) in the network layer. Then, we do the MAC layer scheduling based on the AMC and TMCR.

\section{- Physical layer}

In the PHY layer, what we care about is the transmission quality and the link rate. In the measurementbased deployment, the received signal strength (RSS) is measured for each candidate MR using the path loss model [13] as given in Equation 8. The path loss model describes the attenuation experienced by a wireless signal as a function of distance. The signal power decays exponentially with the distance. Given a reference signal strength $P_{d B m}\left(d_{0}\right)$ at distance $d_{0}$, the RSS at distance $d$ is given as

$$
P_{d B m}(d)=P_{d B m}\left(d_{0}\right)-10 \gamma \log _{10}\left(\frac{d}{d_{0}}\right)+\epsilon,
$$

where $\gamma$ is the path loss exponent, and $\varepsilon$ is the shadowing term.

With a given transmission power, higher rate modulation requires a higher RSS or a shorter transmission distance between two nodes. In order to increase the link capacity while maintaining transmission quality, the AMC technique is used at the physical layer that improves the data transmission rate. To estimate the link rates of the local and the backbone networks, we apply the distance between two nodes using Equation 8 to obtain the RSS first. Then, the RSS is applied into the 802.16 AMC table given in [34] to select an appropriate modulation scheme and thus the corresponding raw bit rate.

\section{- Network layer}

A multi-hop wireless network must have a routing scheme that selects a path to relay packets between IGW and MRs. The shortest path routing and the minimum hop routing (e.g., AODV) are two popular routing schemes. However, different routing schemes are suitable for different networks, such as ad hoc networks, sensor networks, and stationary networks, such as WMNs. Routing has been primarily designed to maintain connectivity for ad hoc networks or sensor networks, whereas it is more important to maximize the network throughput for WMNs.

We define the distance between two links as the shortest distance between two opposite end nodes. As shown in Figure 1, four possible distances between two opposite end nodes of links $l_{j k}$ and $l_{l m}$ are $d(j, l), d(j, m)$, $d(k, l)$, and $d(k, m)$. Then, in this case, the distance between links $l_{j k}$ and $l_{l m}$ equals to $d(k, m)$, i.e., the shortest among the four. Interference occurs when the distance between two links is smaller than the transmission range of an MR. Next, we define the degree of interference for link $l_{j k}$, denoted as $I_{j k}$, as the number of links that are restrained from transmitting because of the interference caused by the transmitting of link $l_{j k}$. As shown in Figure 2, the degree of interference for link $l_{1,2}$ is 5 , since there are five links, i.e., $l_{3,4}, l_{5,6}, l_{7,8}, l_{9,10}$,

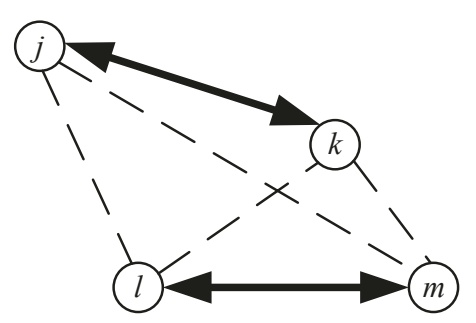

Figure 1 Definition of distance between two links 
and $l_{11,12}$, are interfered by the transmission of link $l_{1,2}$ and thus are restrained from transmission. In Figure 2, the transparent and shaded circles show the transmission ranges of MR 1 and MR 2, respectively. Conversely, link $l_{1,2}$ is restrained from transmission when any one of the interfered links, i.e., $l_{3,4}, l_{5,6}, l_{7,8}, l_{9,10}$, or $l_{11,12}$, is transmitting.

We define the cost of the link between $\mathrm{MR} j$ and MR $k$ as

$$
\operatorname{Cost}_{j k}=I_{j k} / r_{j k}
$$

where $I_{j k}$ is the degree of interference that interfere link $l_{j k}$ and $r_{j k}$ is its link rate. Thus, Cost $t_{j k}$ represents the time duration of interference incurred when transmitting a unit of data over the link $l_{j k}$. The larger $r_{j k}$ is, the shorter will be the transmission time for a data packet, and hence, the shorter the blocking time will be for other links in link $l_{j k}$ 's collision domain. Also, the smaller $I_{j k}$ is, the fewer number of links is interfered by the link $l_{j k}$, and the shorter will be the aggregate blocking time.

In this study, we use a routing scheme, called TMCR, for the backbone relay traffic. TMCR works similar to that in [35], but considers both the capacity and the degree of interference along the path. Thus, TMCR selects a path with the minimum interference capacity. The goal of TMCR is to minimize the aggregate cost along a routing path. We define the routing cost of an MR $l$ as

$$
\operatorname{COST}_{i}=\sum_{l_{j k} \in P_{i}} \operatorname{Cost}_{j k}=\sum_{l_{j k} \in P_{i}}\left(I_{j k} / r_{j k}\right),
$$

where $P_{l}$ represents the routing path from MR $l$ all the way to IGW. Thus, $\operatorname{COST}_{l}$ represents the backbone capacity consumed when a unit of data is transmitted on $P_{l}$.

Algorithm 1 shows the TMCR algorithm. TMCR is a variant of the Prim's algorithm. It finds a minimum spanning tree using a greedy strategy based on $\operatorname{COST}_{l}$. After TMCR terminates, a routing tree $T$ is obtained.

\section{- MAC layer}

It is important to handle all users' demand evenly by nearby MRs. However, since all the internetwork traffic goes through the IGW, MRs closer to the IGW have shorter paths to the IGW and therefore consume less network resource than MRs farer away from the IGW. Thus, we shall give higher priority to MRs closer to the IGW when we assign users' demand to MRs. To achieve this goal, we define a weight-based link assignment (WLA) at the MAC layer. In WLA, we first sort MRs in
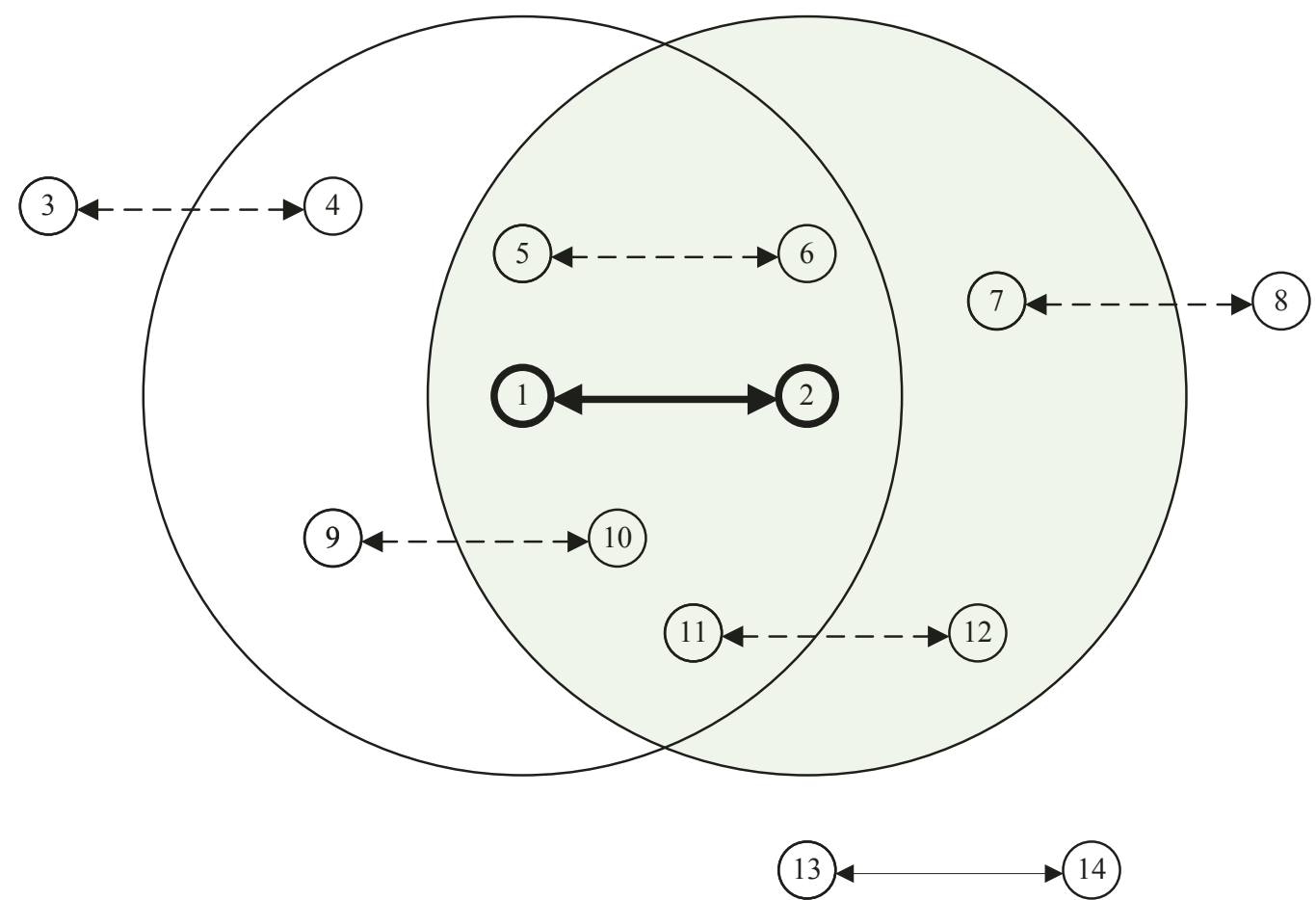

Figure 2 Demonstration of degree of interference for link $I_{1,2}$ 
an increasing order based on their routing cost, as defined in Equation 10. Then, we assign users' demand to MRs according to their order by the nearest neighborhood scheme, i.e., we assign user demand $q_{i}$ to $\mathrm{MR} j$ whose $r_{i j}$ is the largest while guaranteeing such an allocation is supported by the backbone. If the backbone cannot support such a user demand, then WLA terminates, which implies that the scheduling fails. Algorithm 2 shows the procedure for WLA.

As MR is deployed incrementally, the routing tree also changes accordingly. Thus, WLA must be repeated for every MR added. With such a dynamic allocation, we are able to achieve close-to-optimal assignment while ensuring the feasibility of the MRP.

\subsection{Performance metrics}

On the MRP problem, it is hard to solve Equation 1 while satisfying Equations 4 and 5 because of interference. In this subsection, based on the concept of collision domain, we first consider the upper bound for the capacity of a WMN. Then, we introduce two performance metrics: local coverage $(L C)$ and backbone residual capacity $(B R C)$. Using these two metrics, we can quantify the degree of their contribution when deploying an MR both in the users' demand coverage and in the backbone. Then, we present a novel heuristic algorithm based on the metrics for MRP.

\section{- WMN capacity upper bound}

Evaluating the upper bound $C_{w m n}$ for the capacity in a WMN is important for the network planning. It indicates how well users' demand can be satisfied. To estimate $C_{w m n}$, this study utilizes the heuristic of [22] which utilizes the concept of collision domain (CD), and then the most congested CD, called BCD, is identified and used to compute $C_{w m n}$.

A CD covers a set of nodes which should not transmit or receive any data at the same time so as to avoid the mutual interference. To demonstrate how $C_{w m n}$ of a WMN is computed, a chain topology of Figure 3, taken from [22], is used as an example. Here, every node has a demand of $1 G$ to gateway. The CD centered at link 2-3 contains links 1-2, 2-3, 3-4, and 4-5. When the link 2-3 is activated, the links in the 2-3 CD cannot be active at the same time. With similar arguments, we can readily find out CDs of all the links, out of which the CD of link 2-3 contains the most link flows $(4+5+6+7+$ $8) G$ and hence is the BCD of the WMN. If each link in the collision domain of 2-3 cannot forward more than the nominal MAC layer capacity $B$, then the maximal throughput cannot exceed $C_{w m n}=B /(4+5+6+7+8)$ $G=B / 30 G$.

Because all the traffic must be forwarded toward/from the IGW, IGW is the most heavily loaded CD in the network and often becomes the BCD of a WMN [36].
By analyzing the capacity of $\mathrm{BCD}$, we can compute $C_{w m n}$ of a WMN, by which we can decide if the backbone capacity is sufficient to support all the users. The BCD concept holds true for single channel. For multiple channel case, it is easy to iterate for $C_{w m n}$ in a WMN. If each channel has the same characteristics, then it is simply $c \times C_{w m n}$ for $c$ subchannels. However, for simplicity, we assume a single channel case, thereby enabling the use of only $C_{w m n}$.

\section{- Local coverage}

The location of an MR is very important for serving MCs. A user demand is satisfied when both the local and the backbone networks have sufficient capacities to handle. As per PHY layer property, if the distance between two nodes is shorter, then the transmission rate becomes large with AMC. Thus, if we want to enhance the backbone link quality, then we must reduce the transmission distance between the MRs. On the contrary, if we want to serve more MCs, then we should place an MR close to as many uncovered MCs as possible, i.e., extending the transmission distance between MRs. However, the local coverage metric $(L C)$ simply considers serving as many MCs as it can.

The users' demand allocation not only must meet the link and local network constraints, respectively, in Equations 3 and 4, but ought to be supported by the backbone network as well as follows:

$$
\Theta=Q_{I G W}+\sum_{j \in B C D} Q_{j} \leq C_{w m n}
$$

where $Q_{I G W}$ and $Q_{j}$ are the local demands of IGW and MR $j$, respectively. Equation 11 computes the total throughput of the mesh network $\Theta$, which must be smaller than or equal to the network capacity $C_{w m n}$.

When the backbone capacity is large enough to support more users' demand, every newly added MR can cover more users' demand and hence contributes additional throughput. To evaluate the value of a candidate $\mathrm{MR}$, the Local Coverage (LC) metric is used to represent the contribution of an MR in enhancing the network throughput. We define $R_{(n)}$ as an increment to the network throughput when $n^{\text {th }} \mathrm{MR}$ is deployed:

$$
R_{(n)}=\Theta_{(n)}-\Theta_{(n-1)},
$$

where $\Theta_{(n)}$ denotes the throughput of the WMN after the nth MR has been deployed. To determine the $n$th $M R$, the local coverage of every MR, denoted by Equation 4 as $R_{j}$ for $\mathrm{MR} j$, is calculated first. Apparently, it is beneficial to select the MR with the largest LC.

\section{- Backbone residual capacity}

Transmitting data in a wireless multi-hop network consumes substantial resources because of interference among the links. Thus, we must try to cover more 


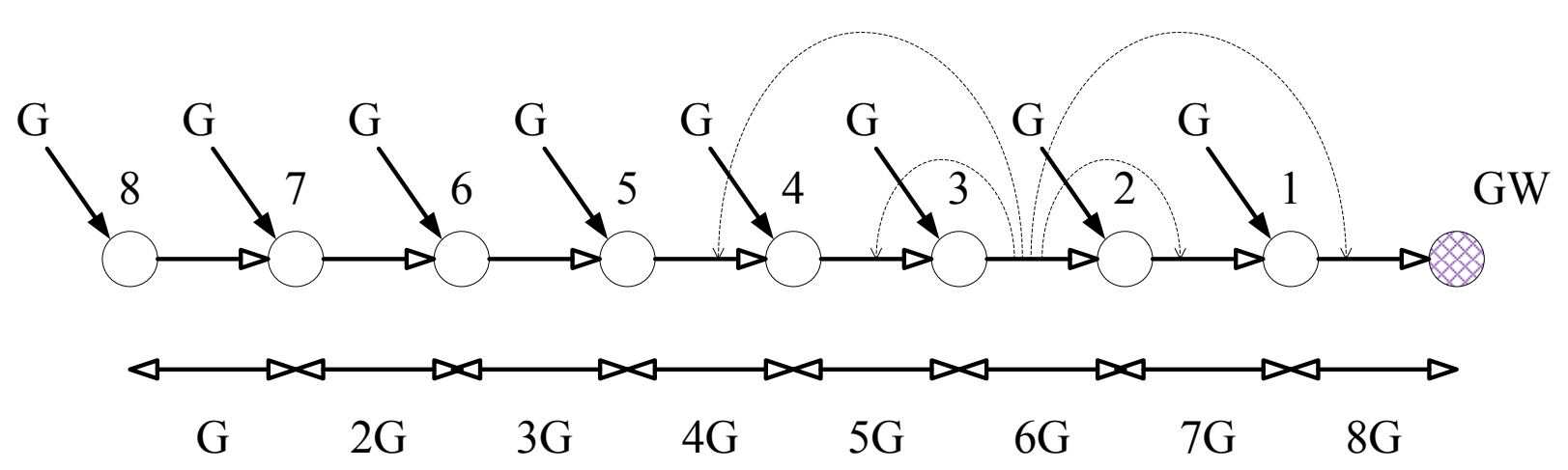

Figure $3 \mathrm{~A}$ case of $\mathrm{BCD}$ in chain topology

users' demand while reducing resource consumption. Because we place MR one by one, it is necessary to compute how much residual resource is available for other unserved users. We define the Backbone Residual Capacity $(B R C)$ metric that estimates the amount of backbone capacity available to serve un-assigned users' demand after placing an MR. BRC computes the residual capacity of all the links in the BCD, i.e., the $\mathrm{CD}$ of the IGW. Because all the data flows must be transmitted through the $B C D$, the resource in the $B C D$ will be exhausted first. Thus, if $B R C$ is larger, then more MCs far away from the IGW can be served.

Algorithm 3 presents the $B R C$ computation algorithm. The residual capacity of link $l_{j k}$, denoted as $\hat{C}_{j k}^{r}$, is its link capacity minus its current aggregated traffic load:

$$
\hat{C}_{j k}^{r}=r_{j k}-\hat{Q}_{j} .
$$

The total residual capacity in the $\mathrm{BCD}$, denoted as $\hat{C}^{r}$, is the sum of the residual capacity of each link in the BCD:

$$
\hat{C}^{r}=\sum_{j k \in B C D} \hat{C}_{j k}^{r}=\sum_{j k \in B C D}\left(r_{j k}-\hat{Q}_{j}\right)
$$

where $l_{j k}$ means MR $k$ is the uplink node of MR $j$. We denote $\hat{C}_{j}^{r}$ as the total residual capacity if $\mathrm{MR} j$ is deployed.

When $\hat{C}^{r}$ is zero while some users' demand are still un-assigned, the WMN design either fails or omnidirectional antennas for some MRs must be replaced by directional antennas so as to reduce the interference, and thus the link rate could be increased.

\section{Cross-layer MRP}

Using two performance metrics and the cross-layer design described above, we introduce the first heuristic algorithm, named CMRP-1, to efficiently place MRs in a
WMN. In CMRP-1, we choose a candidate MR $k$ to maximize the objective function $O F_{1}$ as follows:

$$
k=\arg \max _{j}\left\{O F_{1}(j)\right\},
$$

where

$$
O F_{1}(j)=B R C_{j} \times R_{j}
$$

Instead of selecting $M R$ with a maximal $B R C$ or a maximal $L C$, we select $M R$ with the maximum product of $B R C$ and $L C$ first. The reason we select $B R C \times L C$ is to maximize the backbone capacity while covering more users' demand. If only $L C$ is used, then the MR deployment will always select an MR with maximal user demand coverage, which can cover substantial users' demand initially, but completely consume the backbone resource soon, resulting in a non-optimal placement. Thus, the product of $B R C$ and $L C$ can allow us to balance the effectiveness between the coverage of users' demand and improvement in the backbone capacity.

Based on $O F_{1}$, Algorithm 4 contains three main parts. The first part is to select an IGW location, the second part is to deploy an MR, and the last part is to deploy directional antennas for backbone links.

Step 1 initializes the routing tree $T$ using TMRC as given in the Algorithm 1. Step 3 determines the IGW location. The IGW deployment problem is beyond the scope of this article. So, we use an existing approach given in [9] to select the IGW location. In Step 6, we calculate all the candidate MR locations to find a location with the maximum $O F_{1}$ and deploy it. In Steps 10 and 11 , we reconstruct the scheduling routing tree and re-allocate users' demand with TMRC and WLA, respectively. If we cannot find an $\mathrm{MR}$ location with $O F_{1}$ $>0$, then it implies that the backbone capacity is exhausted and cannot satisfy any more demand. However, there may be some links that could be split by another MR to enhance their link rates and hence 
increase the backbone throughput. Thus, we temporarily ignore $L C$ and select an MR with a maximal average $B R C$, denoted as Max_R, larger than a threshold and deploy it. If no such MR exists, then Step 20 finds a link that interferes with other links for the longest time period and replaces it with a pair of directional antennas. This algorithm will be terminated either when all the users' demands are satisfied or when we cannot deploy an MR at a new location to increase the backbone throughput any further.

In CMRP-1, directional antennas are used only when the WMN topology cannot meet all users' demand and the MR locations are not changed when directional antennas are added. Because using directional antennas not only reduces the interference, but also enhances the transmission range, such a deployment scheme may not be optimal. Furthermore, CMRP-1 does not consider the deployment cost of an MR and an antenna and cannot optimize the cost of the WMN deployment.

To cope with the weakness of CMRP-1, we define a Deployment Cost (DC) metric as an index to estimate the cost of using directional antennas. The cost of deploying MR $j$ using directional antennas, denoted as $D C_{j}$, is defined as

$$
D C_{j}=\frac{\alpha_{j}+\beta_{j k}}{\alpha_{j}}=1+\frac{\beta_{j k}}{\alpha_{j}},
$$

where $\beta_{j k}$ is the cost increase of using a pair of directional antennas between MR $j$ and its uplink MR $k$, and $\alpha_{j}$ is the cost of deploying MR $j$ using omni-directional antenna only. Then, the second objective function is defined as

$$
\mathrm{OF}_{2}(j)=B R C_{j} \times R_{j} / D C_{j} .
$$

The CMRP-1 is then revised to be CMRP-2 that always chooses a candidate MR $k$ where

$$
k=\arg \max _{j}\left\{O F_{1}(j), O F_{2}(j)\right\} .
$$

If MR $k$ is selected and its $O F_{2}$ is larger than its $O F_{1}$, then MR $k$ will use a pair of directional antennas on the link between itself and its parent node in the routing tree. We denote $\beta / \alpha$ in Equation 17 as the cost ratio (CR). Lower cost of directional antenna means smaller CR, i.e., $D C$ is closer to unity and $O F_{1} \approx O F_{2}$, and thus CMRP-2 performs nearly as CMRP-1. The cost of deploying a new MR is always there. What we consider in $C R$ is the difference between the cost of adopting directional antenna and omni-directional antenna. We show how CR affects CMRP-2 in Section 5.

The complexities of TMCR, WLA, and BRC are $O$ $\left(m^{2}\right), O\left(m^{2}\right)+O(m \times n \log n)$, and $O\left(m^{2}\right)$, respectively. The complexity of CMRP is thus $O\left(\mathrm{~m}^{3}\right)+O$ $\left(m^{2} \times n \log n\right)$.

\section{The algorithm simulation and analysis}

Using the proposed heuristic algorithm, we evaluate the cost of deploying an IEEE 802.16d WMN with only one IGW. Users are randomly distributed in the area under consideration. In the network, we use TDMA technology. Table 1 indicates the parameters used in the simulation. The interference range is set to be twice the transmission range. All the programs for our simulation are written in C++ and built by Microsoft Visual Studio 2005.

\subsection{Comparing with another algorithm}

We compare our algorithms, CMRP-1 and CMRP-2, with another Probability algorithm proposed in [13], which is similar to our approach among other related works, i.e., it also uses a heuristic algorithm that places mesh nodes one by one while keeping an eye on the local coverage and the backbone connectivity probability. In our simulation, 180 users and 180 candidate MR locations are configured in a square of $6 \mathrm{~km}$. Each user has 1.0 Mbps uplink flow demand.

The simulation result shown in Figure 4 illustrates that the Probability cannot produce the maximum network throughput, i.e., 180 Mpbs, until the 107th MR is deployed. This is because the algorithm is not designed to maximize the network throughput, and thus it cannot let a network adapt well to large users' demand. However, CMRP-1 and CMRP-2 can reach the maximum network throughput with only 33 MRs and 2 pairs of directional antennas, and 20 MRs and 10 pairs of directional antennas, respectively. CMRP-2 can provide the maximum network throughput with the minimum deployment cost and with less computation time. Assume $C R=0.3$. Then, Figure 5 shows the CPR vs. network throughput. When the network throughput is low (e.g., below $65 \mathrm{Mbps}$ ), all the three algorithms perform equally well. But, when a larger network throughput (e.g., over $65 \mathrm{Mbps}$ ) is desirable, the CPR for the Probability reduces rapidly. When the network throughput is increased further (e.g., over $140 \mathrm{Mbps}$ ), the CPR

\section{Table 1 The setting of parameters in the PHY layer}

\begin{tabular}{lc}
\hline Local power & $\mathbf{0 . 0 1} \mathbf{~ W}$ \\
Backbone omni-directional antenna power & $0.5 \mathrm{~W}$ \\
Backbone directional antenna power & $0.8 \mathrm{~W}$ \\
Backbone directional antenna angle & $30^{\circ}$ \\
Local path loss & 3.8 \\
Backbone path loss & 3.6 \\
Local transmission range & $1050 \mathrm{~m}$ \\
Backbone transmission range & $2100 \mathrm{~m}$ \\
Backbone transmission range with directional antenna & $2500 \mathrm{~m}$ \\
Local link bandwidth & $10 \mathrm{Mbps}$ \\
Backbone link bandwidth & $30 \mathrm{Mbps}$ \\
\hline
\end{tabular}




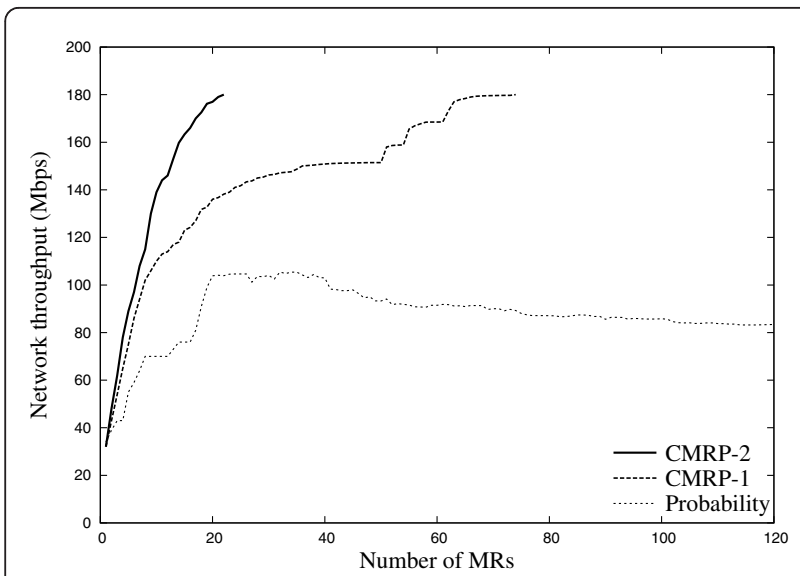

Figure 4 Network throughput vs. number of MRs for the MR deployment by CMRP-2, CMRP-1, and Probability

for CMRP-1 becomes worse than CMRP-2. Thus, we conclude that CMRP-2 has the best CPR for all the ranges of the network throughput.

\subsection{Comparing CMRP with fair scheduling, shortest path routing, and greedy MR selection method}

In order to show the merit of CMRP-1 and CMRP-2, we first compare the simulation results of the CMRP framework with various existing schemes, such as the fair user demand allocation, the shortest path routing, and a greedy MR selection scheme, denoted as CMRP-1/Fair, CMRP-1/SP, and CMRP/Greedy, respectively. The fair user demand allocation scheme assigns users' demand to MRs solely based on the nearest neighborhood scheme, without considering the locations of MRs relative to the IGW. The shortest path routing scheme constructs the smallest hop count routing paths between IGW and MRs without considering the link rate and the interference. The greedy MR selection scheme always

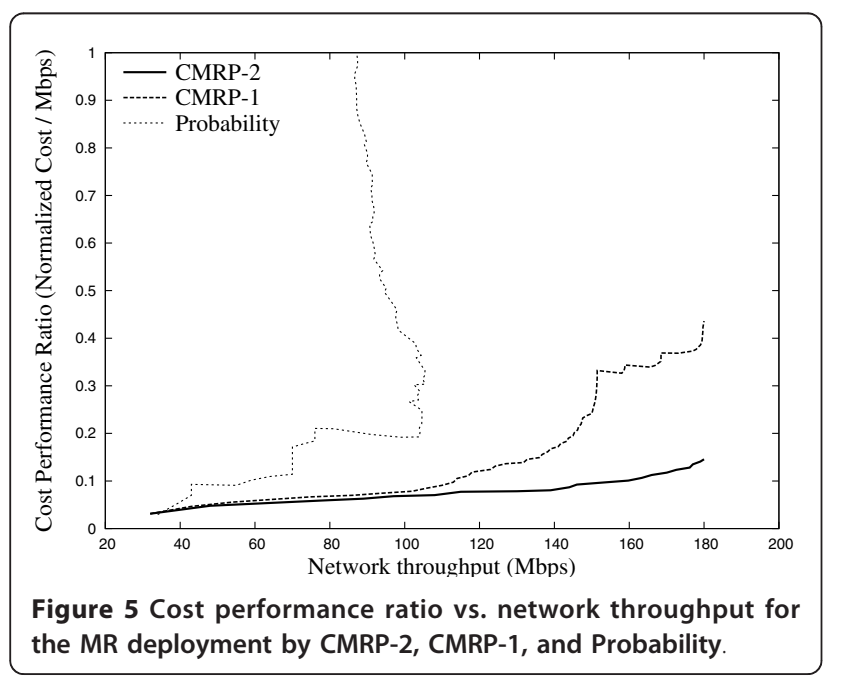

chooses an MR with the best throughput based on the $L C$ only.

The testing environment is the same as discussed earlier in Section 5.1, except that the user demand is varied from 0.6 to 1.5 Mbps. We run 100 simulations with randomly generated scenarios on each scheme and retain only those successful results that satisfy all users' demand. Table 2 shows the percentage of simulation failure for each scheme. The result shows that CMRP-1/ SP collapses at larger users' demand and performs the worst among all the schemes. Based on only a few successful simulations, the simulation results of CMRP-1/ SP for higher users' demand become unreliable and thus are omitted in Figures. 6,7,8 and 9. Table 2 also shows that CMRP-1 outperforms CMRP-1/Fair when the users' demand becomes large, which substantiates that WLA performs better than that of the fair user allocation scheme. The success rate of CMRP-2 is smaller than that of CMRP-1 and CMRP/Greedy because CMRP-2 deploys directional antennas along with MRs, which makes the addition of directional antennas less useful in augmenting BRC.

Figures 6 and 7 show the number of MRs and the number of pairs of directional antennas deployed by each scheme. Figure 6 shows that CMRP-2 deploys the fewest MRs and the number of MRs deployed by CMRP-2 is relatively independent of the users' demand. Figure 7 shows that the number of pairs of directional antennas increases as users' demand increases for all the schemes. However, the number of pairs of directional antennas deployed by CMRP-2 is linearly dependent on the demand. This shows that taking the antenna type into account while deploying MRs is an efficient way to minimize deployment cost.

\subsection{Analyzing the cost of constructing a WMN}

As the cost is an important index to determine how good an MR deployment algorithm is for service providers, we discuss the cost of constructing a WMN. Figure 8 shows the no rmalized cost of all the schemes relative to CMRP/Greedy. It is shown that CMRP-2 achieves the lowest deployment cost among all the schemes, and the CPR is the lowest as the user demand increases up to 1.0 Mbps. The result also shows that the deployment schemes without considering cost converge as the user demand increases. Figure 9 shows that CMRP-2 provides the least CPR and is nearly constant for all the ranges of user demand, while the CPR of other schemes increases as the user demand increases. This shows that CMRP-2 is much more cost effective and efficient in the MR deployment.

Figure 10 shows CPR vs. user demand for various CRs. It is shown that CPR slightly increases as the users demand increase. Also, CPR increases as CR 
Table 2 Percentage of simulation failure by different schemes

\begin{tabular}{lccccc}
\hline Demand & CMRP-2 (\%) & CMRP-1 (\%) & CMRP-1/Fair (\%) & CMRP-1/SP (\%) & CMRP/Greedy (\%) \\
\hline 0.6 & 0 & 0 & 0 & 0 & 0 \\
0.7 & 7 & 0 & 0 & 0 & 0 \\
0.8 & 5 & 0 & 0 & 97 & 0 \\
0.9 & 12 & 0 & 0 & 98 & 0 \\
1.0 & 18 & 0 & 0 & 99 & 0 \\
1.1 & 19 & 0 & 7 & 100 & 0 \\
1.2 & 21 & 6 & 12 & 100 & 12 \\
1.3 & 23 & 9 & 30 & 100 & 15 \\
1.4 & 17 & 60 & 100 & 32 \\
1.5 & 46 & 38 & & \\
\hline
\end{tabular}

increases, and the deployment cost is relatively stable when $C R$ is small in various user demands. Figure 11 shows CPR vs. total deployment cost (MRs plus directional antennas) for various CRs. As shown in Figure 11 , there is a sharp increase in CPR for each CR, which indicates large deployment cost over small throughput obtained and thus the optimal bound for deployment cost. This information is useful when we plan a large-scale WMN with more than one IGW in optimizing deployment cost.

\section{Conclusion and future work}

In this article, we present a CMRP scheme for IEEE 802.16d WMNs. CMRP integrates the AMC technology and the antenna type at the PHY layer, TMCR at the network layer, MAC scheduling and WLA at the data link layer to derive a cost effective WMN design. CMRP encapsulates the complex design metrics into three design attributes: the local coverage, the backbone residual capacity, and the deployment cost. Numeric results show that CMRP works well, and provides a good CPR in the WMN network planning. Simulation results also confirm that our novel TMCR and WLA schemes can

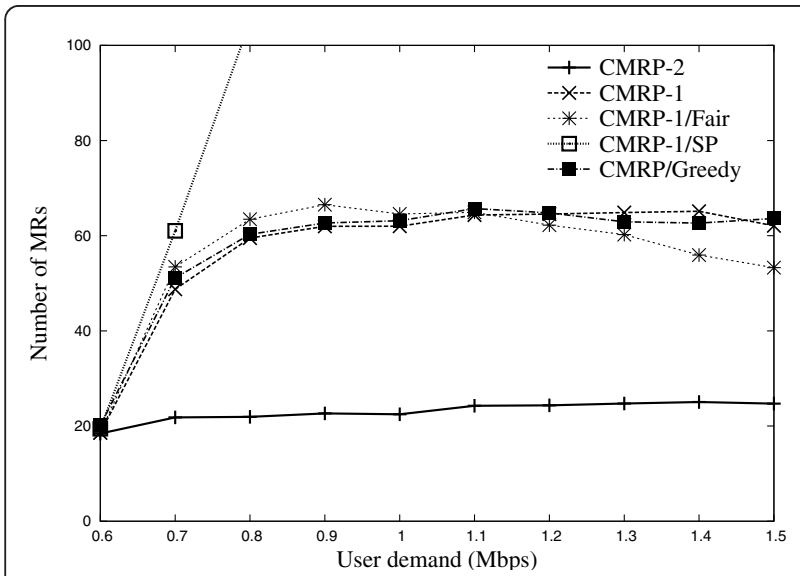

Figure 6 Number of mesh routers by various schemes. effectively improve the performance of CMRP. Moreover, by incorporating the cost ratio of directional antenna to MR in the network planning, a WMN with a low CPR can be obtained.

From the simulation results, we also see that the CPR increases substantially as a WMN covers larger users' demand. Based on this observation, we plan to develop an IGW placement algorithm based on CMRP to achieve low CPR in the large-scale WMN planning.

\section{Algorithm 1 TMCR}

Input: backbone topology $G=\left(V^{\prime}, E\right)$, link rates $r_{j k \mid j k \in E}$, and IGW.

Output: a routing tree $T$.

$/ / M_{c}$ : the set of MR candidates that have not yet been

$/ /$ included in a routing tree.

$/ / M_{r}$ : the set of MR candidates that have

been

$$
\text { // included in a routing tree. }
$$

1: $M_{c}=V-\{I G W\}$;

2: $M_{r}=\{I G W\} ; T=\{I G W\} ; C O S T_{I G w}=0$;

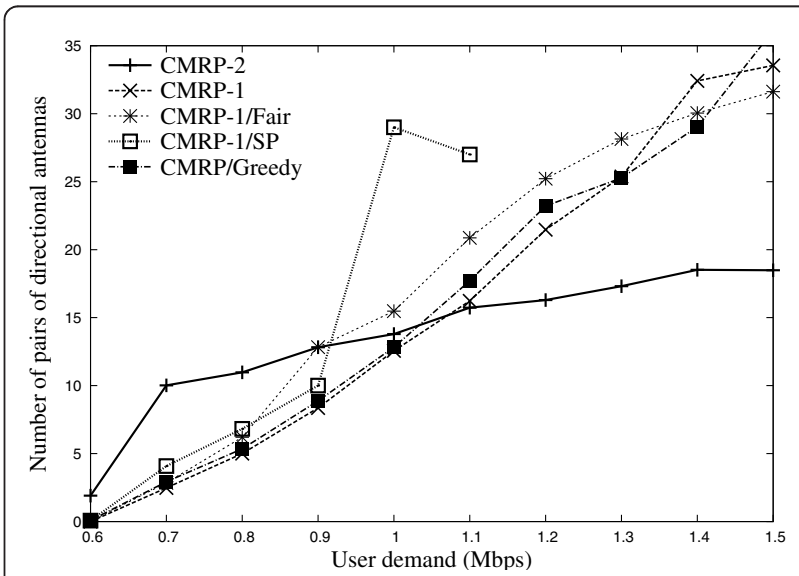

Figure 7 Number of pairs of directional antennas by various schemes. 


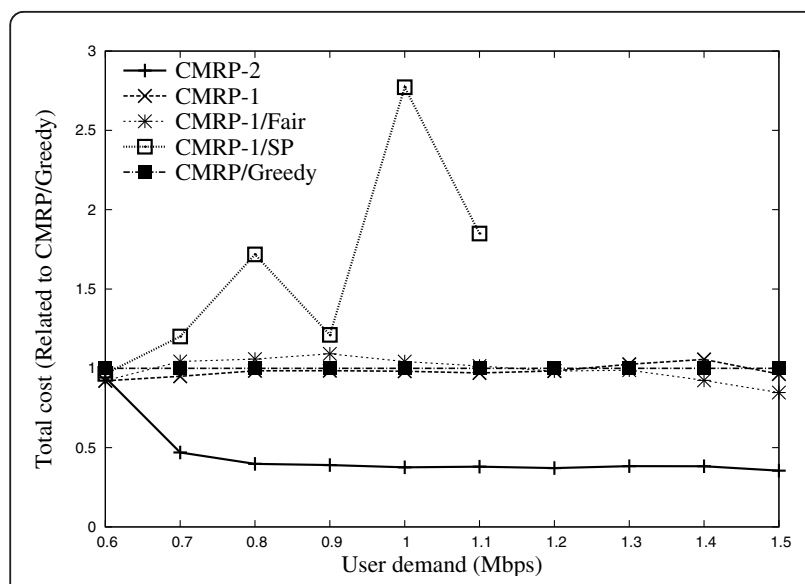

Figure 8 Normalized total cost by various schemes.
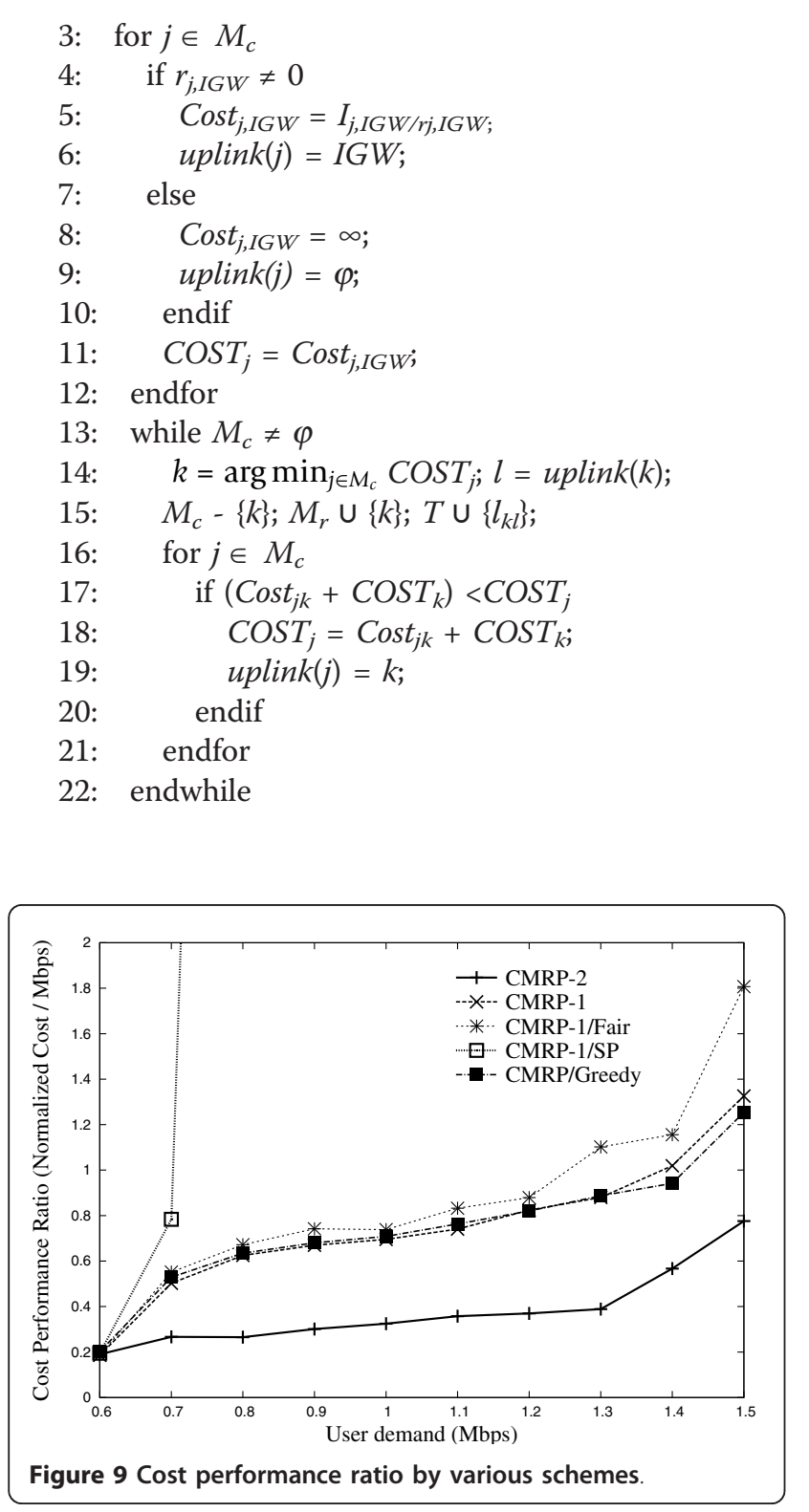

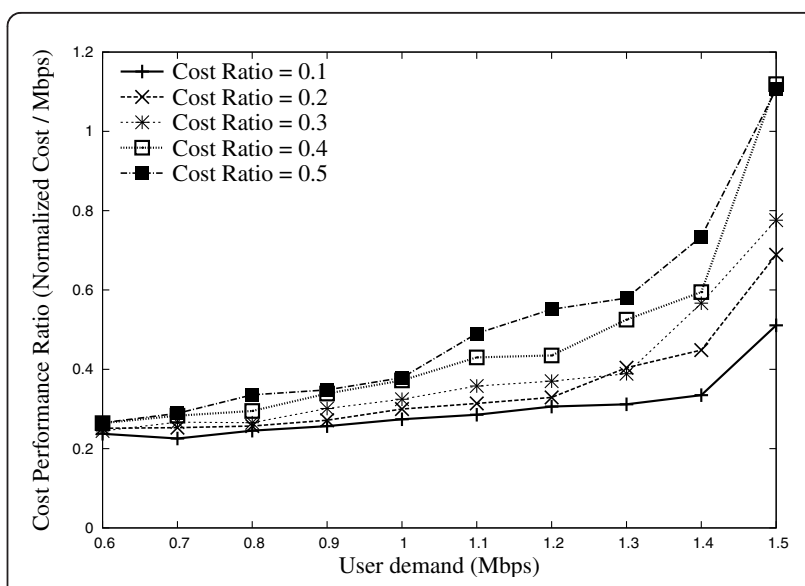

Figure 10 Cost performance ratio vs. user demands for CMRP2 .

\section{Algorithm 2 WLA}

Input: a routing tree $T$, MR routing costs $C O S T_{j \mid j \in M_{r}}$, users' demand $\left.q_{i}\right|_{i} V$.

Output: the user's demand allocation $A$.

1: $S=M_{r} ; D=\left\{q_{i} \mid i \in V\right\}$

2: $\quad$ flag $=1$;

3: while $S \neq \varphi$ \&\&flag $==1$

4: $\quad k=\arg \min _{j \in S} \operatorname{COST} T_{j}$;

5: $\quad$ sort $q_{i}$ in $D$ in a descending order of $r_{i k}$;

6: $\quad R_{k}=R_{L}$;

7: $\quad$ while $D \neq \varphi$ and $R_{k}$ is not exhausted

8: $\quad$ assign the first $q_{i}$ in $D$ to $A(k)$;

9: $\quad D-\left\{q_{i}\right\} ; R_{k}=R_{k}-q_{i}$;

10: $\quad$ allocate backbone resource to $q_{i}$;

11: $\quad$ if backbone resource is exhausted

12: $\quad$ flag $=0$; break;

13: $\quad$ endif

14: endwhile

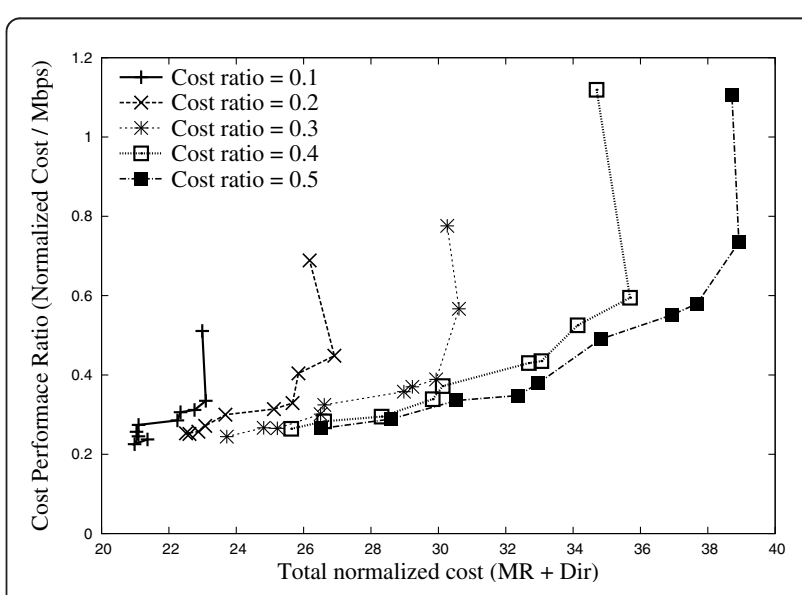

Figure 11 Cost performance ratio vs. total normalized cost for CMRP-2. 
15: $\quad S-\{k\}$;

16: endwhile

\section{Algorithm 3 Backbone Residual Capacity (BRC) computation}

Input: the set of scheduled backbone links $L_{B}$.

Output: the backbone residual capacity $\hat{C}^{r}$.

$$
/ / \hat{C}_{j k}^{r} \text { : the residual capacity of link } l_{j k}
$$

1: $\quad U=L_{B}$

2: $\quad \hat{C}^{r}=0$;

3: while $U \neq \varphi$

4: $\quad$ select a link $l_{j k}$ from $U$;

5: $\quad U-\left\{l_{j k}\right\}$;

6: $\quad$ if $l_{j k}$ is in the BCD

7: $\quad \hat{C}_{j k}^{r}=r_{j k}-\hat{Q}_{j}$;

8: $\quad \hat{C}^{r}=\hat{C}^{r}+\hat{C}_{j k^{i}}^{r}$

9: $\quad$ endif

10: endwhile

\section{Algorithm 4 CMRP-1}

Input: user location $V$, demand $q_{i \mid i \in V}$, MR candidate locations $M_{c}$, and IGW location $I G W$.

Output: the number of selected MRs and their respective locations $M_{r}$.

1: initialize the routing tree $T$ by TMCR;

2: $\quad M_{r}=\{I G W\}$

3: set up IGW location;

4: $M_{c}-\{I G W\}$;

5: while $\Theta<\sum_{i=1}^{n} q_{i}$

6: $\quad \mathrm{j}=\arg \max _{M c}\left\{O F_{1}>0\right\}$;

7: $\quad$ if $j \neq-1 / /$ current backbone capacity $/ /$ is large enough

8: $\quad M_{c}-\{j\}$;

9: $\quad M_{r} \cup\{j\}$

10: $\quad$ update the routing tree $T$ by TMCR;

11: reallocate users' demand by WLA;

12: $\quad$ else $/ / j=-1$

13: $\quad$ find a location $k \in M_{c}$

with $M a x \_R=\max _{k \in M_{r}}\left\{B R C_{k} /\left|M_{r}\right|\right\}$;

14: $\quad$ if $\left(\operatorname{Max} \_R>\omega\right) / / \omega$ is a threshold

15: $\quad M_{\mathrm{c}}-\{k\}$;

16: $\quad M_{\mathrm{r}} \cup\{k\}$;

17: $\quad$ update the routing tree $T$ by TMCR;

18: $\quad$ reallocate users' demand by WLA;

19: $\quad$ else// no MR can be selected from $M_{c}$

$$
\text { //with Max_R> } \omega
$$

20:

find a link $l_{i j} \in B C D$

$$
\text { with maximal } \frac{Q_{i}}{r_{i j}} \times I_{i j}
$$

21: if no such a link is found

22:

23:

24:

antennas;

25: $\quad$ update the routing tree $T$ by TMCR;

26:

27:

28:

29.

$$
\text { endif }
$$$$
\text { endif }
$$

endwhile

endif

update the routing tree $T$ by TMCR;
reallocate users' demand by WLA;

dif replace $l_{i j}$ by a pair of directional

\section{Acknowledgements}

The authors would like to thank the National Science Council, Taiwan, ROC, for financially supporting this research under Contract No. NSC100-2221-E155-040. The authors would also like to thank Prof. Dharma P. Agrawal for his precious comment and suggestion on this research work.

\section{Author details}

${ }^{1}$ Yuan-Ze University, Chung-Li, Tao-Yuan, Taiwan ${ }^{2}$ Oriental Institute of Technology, Banqiao District, New Taipei City, Taiwan

\section{Competing interests}

The authors declare that they have no competing interests.

Received: 1 March 2011 Accepted: 14 October 2011

Published: 14 October 2011

References

1. IF Akyildiz, X Wang, A survey on wireless mesh networks. IEEE Commun Mag. 43(9), S23-S30 (2005)

2. N Nandiraju, D Nandiraju, L Santhanam, B He, J Wang, DP Agrawal, Wireless mesh network: current challenges and future directions of web-in-the-sky. IEEE Wireless Commun Mag. 14(4), 79-89 (2007)

3. Nortel [Online], http://www.nortel.com

4. Tropos Networks [Online], http://www.tropos.com

5. Strix Systems [Online], http://www.strixsystems.com

6. BelAir Networks [Online], http://www.belairnetworks.com

7. A So, B Liang, Enhancing WLAN capacity by strategic placement of tetherless relay points. IEEE Trans Mobile Comput. 6(5), 474-487 (2007)

8. MR Souryal, J Geissbuehler, LE Miller, N Moayeri, Real-time deployment of multihop relays for range extension, in Proceedings of the ACM International Conference on Mobile Systems, Applications, and Services, 85-98 (2007)

9. J Wang, B Xie, K Cai, DP Agrawal, Efficient mesh router placement in wireless mesh network, in Proceedings of the IEEE International Conference on Mobile Adhoc and Sensor Systems (2007)

10. S Sen, B Raman, Long distance wireless mesh network planning: problem formulation and solution, in Proceedings of the ACM International Conference on World Wide Web, 893-902 (2007)

11. A So, B Liang, Optimal placement of relay infrastructure in heterogeneous wireless mesh networks by Bender's decomposition, in Proceedings of the ACM International Conference on Heterogeneous Networking for Quality, Reliability, Security and Robustness, a20-a23 (2006)

12. J Robinson, EW Knightly, A performance study of deployment factors in wireless mesh networks, in Proceedings of the IEEE International Conference on Computer Communications, 2054-2062 (2007)

13. AA Franklin, CSR Murthy, Node placement algorithm for deployment of two-tier wireless mesh networks, in Proceedings of the IEEE Global Communications Conference, 4823-4827 (2007)

14. F Xhafa, C Sanchez, L Barolli, Locals search algorithms for efficient route nodes placement in wireless mesh networks, in Proceedings of the IEEE 
International Conference on Network-Based Information Systems, 572-579 (2009)

15. F Xhafa, C Sanchez, L Barolli, Genetic algorithms for efficient placement of router nodes in wireless mesh networks, in Proceedings of the IEEE International Conference on Advanced Information Networking and Applications, 465-472 (2010)

16. F Xhafa, C Sanchez, L Barolli, R Miho, An annealing approach to router nodes placement problem in wireless mesh networks, in Proceedings of the IEEE International Conference on Complex, Intelligent and Software Intensive Systems, 245-252 (2010)

17. J Wang, K Cai, DP Agrawal, A multi-rate based router placement scheme for wireless mesh networks, in Proceedings of the IEEE International Conference on Mobile Adhoc and Sensor Systems, 100-109 (2009)

18. J Yuan, Z Li, W Yu, B Li, A cross-layer optimization framework for multihop multicast in wireless mesh networks. IEEE J Sel Areas Commun. 24(11), 2092-2103 (2006)

19. S Kim, X Wang, M Madihian, Cross-layer design of wireless multihop backhaul networks with multiantenna beamforming. IEEE Trans Mobile Comput. 6(11), 1259-1269 (2007)

20. M Cao, X Wang, S-J Kim, M Madihian, Multi-hop wireless backhaul networks: a cross-layer design paradigm. IEEE J Sel Areas Commun. 25(4), 738-748 (2007)

21. SH Ali, KD Lee, VCM Leung, Dynamic resource allocation in OFDMA wireless metropolitan area networks [Radio Resource Management and Protocol Engineering for IEEE 802.16]. IEEE Wireless Commun Mag. 14(1), 6-13 (2007)

22. J Jun, ML Sichitiu, The nominal capacity of wireless mesh networks. IEEE Wireless Commun Mag. 10(5), 8-14 (2003). doi:10.1109/MWC.2003.1241089

23. KD Lee, VCM Leung, Fair allocation of subcarrier and power in an OFDMA wireless mesh network. IEEE J Sel Areas Commun. 24(11), 2051-2060 (2006)

24. J Tang, G Xue, C Chandler, W Zhang, Link scheduling with power control for throughput enhancement in multihop wireless networks. IEEE Trans Vehicular Technol. 55(3), 733-742 (2006). doi:10.1109/TVT.2006.873836

25. J Bicket, J Bicket, D Aguayo, S Biswas, R Morri, Architecture and evaluation of an unplanned $802.11 \mathrm{~b}$ mesh network, in Proceedings of the ACM International Conference on Mobile Computing and Networking, 31-42 (2005)

26. AD Amis, R Prakash, THP Vuong, DT Huynh, Max-min D-cluster formation in wireless ad hoc networks, in Proceedings of the IEEE International Conference on Computer Communications, 32-41 (2000)

27. S Banerjee, S Khuller, A clustering scheme for hierarchical control in multihop wireless networks, in Proceedings of the IEEE International Conference on Computer Communications, 1028-1037 (2001)

28. JL Wong, R Jafari, M Potkonjak, Gateway placement for latency and energy efficient data aggregation, in Proceedings of the IEEE Conference on Local Computer Networks, 490-497 (2004)

29. Y Bejerano, Efficient integration of multihop wireless and wired networks with QoS constraints. IEEE/ACM Trans Networking. 12(6), 1064-1078 (2004). doi:10.1109/TNET.2004.838599

30. R Chandra, L Qiu, K Jain, M Mahdian, Optimizing the placement of internet TAPs in wireless neighborhood networks, in Proceedings of the IEEE International Conference on Network Protocols, 271-282 (2004)

31. B Aoun, R Boutaba, Y Iraqi, G Kenward, Gateway placement optimization in wireless mesh networks with QoS constraints. IEEE J Sel Areas Commun. 24(11), 2127-2136 (2006)

32. B He, B Xie, DP Agrawal, Optimizing the internet gateway deployment in a wireless mesh network, in Proceedings of the IEEE International Conference on Mobile Adhoc and Sensor Systems (2007)

33. AC Talay, A gateway access-point selection problem and traffic balancing in wireless mesh networks. Springer Lect Notes Comput Sci. 4448, 161-168 (2007). doi:10.1007/978-3-540-71805-5_18

34. IEEE 802.16 - Standard for local and metropolitan area networks, Part 16: Air Interface for Broadband Wireless Access Systems (2004)

35. H-Y Wei, S Ganguly, R Izmailov, ZJ Haas, Interference-aware IEEE 802.16 WiMax mesh networks, in Proceedings of the IEEE Vehicular Technology Conference, 3102-3106 (2005)

36. T-YD Chung, K-C Lee, H-CG Lee, A novel capacity analysis for wireless backhaul mesh networks, in Proceedings of the IEEE Wireless Communication and Networking Conference, 2343-2348 (2008)

doi:10.1186/1687-1499-2011-134

Cite this article as: Chung et al: A novel cross-layer mesh router placement scheme for wireless mesh networks. EURASIP Journal on Wireless Communications and Networking 2011 2011:134.

\section{Submit your manuscript to a SpringerOpen ${ }^{\mathcal{O}}$ journal and benefit from:}

- Convenient online submission

- Rigorous peer review

- Immediate publication on acceptance

- Open access: articles freely available online

- High visibility within the field

- Retaining the copyright to your article

Submit your next manuscript at $\boldsymbol{~ s p r i n g e r o p e n . c o m ~}$ 